\title{
STRONGLY HEREDITARY RADICALS
}

\author{
W. G. LEAVITT
}

A radical class $P$ of some universal class $W$ of not necessarily associative rings is hereditary if $R \in P$ implies $I \in P$ for all ideals $I$ of $R$, and will be called strongly hereditary (terminology suggested by M. Slater) if for all $R \in W$ we have $\rho(I)=I \cap \rho(R)$ for all ideals $I$ of $R$. (Note that in [2] it is the classes with this last property which are called "hereditary".) It is well known that these properties are equivalent if $W$ is any universal class of associative or alternative rings [1, Lemma 1, p. 595 together with Corollary 1, p. 597 for associative rings or Corollary 1, p. 602 for alternative rings]. It is also clear that every strongly hereditary class is hereditary, but the converse is not true in general. This was shown by E. P. Armendariz in [2] using either of the rings of [3, p. 1116]. Note that by [1, Lemma 2 , p. 595] hereditary radical classes are strongly hereditary if their semisimple classes are also hereditary.

Since the hereditary and strongly hereditary properties are in general distinct, E. P. Armendariz has raised the question (private communication) of the existence of a strongly hereditary radical construction analogous to the lower radical. The key to such a construction is provided by the following alternative criterion for a strongly hereditary radical:

THEOREM 1. A hereditary radical class $\beta$ of a universal class $W$ is strongly hereditary if and only if it has property:

(a) If $J \in P$ is an ideal of an ideal I of some $R \in W$ then $J^{\prime} \in P$ where $J^{\prime}$ is the ideal of $R$ generated by $J$.

Proof. Suppose first that $\odot$ is strongly hereditary, then $\odot(I)$ is an ideal of $R$. Thus $J \in P$ implies $J \subseteq P(I)$ so that $J^{\prime} \subseteq \mathcal{P}(I)$ and since $\odot$ is hereditary it follows that $J^{\prime} \in \mathcal{P}$. On the other hand, suppose $\odot$ satisfies condition (a). Let $I$ be an ideal of an $R \in W$ and suppose $J \in \mathcal{P}$ is an ideal of $I$. Then $J \subseteq J^{\prime} \in \mathcal{P}$ and since $J^{\prime}$ is an ideal of $R$ we have $J^{\prime} \subseteq \odot(R)$. Thus $\rho(I) \subseteq I \cap \rho(R)$. But the reverse inequality follows from $P$ hereditary, and thus $P$ is strongly hereditary.

We now proceed to construct a minimal strongly hereditary radical class. We will use the hereditary closure $[3$, p. 1114] of a class $\mathfrak{N}$, namely

Received by the editors October 8, 1968. 
(1) $\mathscr{g} \mathscr{T}=U_{n} \mathscr{G}_{n} \mathscr{T}$, where $\mathscr{I}_{1}(\mathfrak{T})=\{K \mid K$ is an ideal of some $R \in \mathscr{T}\}$ and $\mathscr{I}_{n+1} \mathfrak{T l}=\mathscr{g}_{1}\left(\mathscr{I}_{n} \mathfrak{T}\right)$.

We will also write for a radical class $P$ of a universal class $\%$,

(2) $\mathcal{S} P=\{K \in W \mid J$ is an ideal of an ideal $I$ of some $R \in W$, with $J \in \mathcal{P}$ and $K$ the ideal of $R$ generated by $J\}$.

Note that (trivially) $P \subseteq \mathcal{G}$. Also remark that for an arbitrary class $\mathfrak{T}$ we write $\mathfrak{L} \mathscr{T}$ for the lower radical class defined by $\mathfrak{T}$.

THEOREM 2. Every hereditary radical class $\beta$ of any universal class $W$ is contained in a unique minimal strongly hereditary radical class.

Proof. We proceed by transfinite induction, starting with $\mathfrak{H}_{1}=\varnothing$ and, as usual, if $\beta$ is a limit ordinal $\mathfrak{H}_{\beta}=\mathrm{U}_{\alpha<\beta} \mathfrak{H}_{\alpha}$. On the other hand if $\beta-1$ exists, define $\mathfrak{H C}_{\beta}=\mathcal{L S G G C}_{\beta-1}$. Then write $\mathfrak{H}=\bigcup \mathfrak{H}_{\beta}$ taken over all ordinals $\beta$. It is clear that $\mathfrak{F}_{\beta-1} \subseteq \mathfrak{F}_{\beta}$ and, by induction, $\alpha<\beta$ implies $\mathfrak{H}_{\alpha} \subseteq \mathfrak{F}_{\beta}$. Each $\mathfrak{F}_{\beta}$ is the $\mathscr{L}$ of some hereditary class and so by [4] is a hereditary radical class. Thus $\mathcal{H}$ is hereditary. Clearly $\mathfrak{H}$ is homomorphically closed since this is true of each $\mathfrak{H}_{\beta}$, and suppose we have some $R \in W$ such that every nonzero image $R / I$ contains a nonzero ideal $Q / I \in \mathcal{H}$. Then $Q / I \in \mathcal{H}_{\alpha}$ for some $\alpha$ and, since the collection of all such images is a set, it follows that there is an ordinal $\gamma$ exceeding all such $\alpha$. Thus all $Q / I \in \mathcal{H}_{\gamma+1}=\mathscr{L g G H C}_{\gamma}$ and so $R \in \mathfrak{H}_{\gamma+1} \subseteq \mathcal{H}$. Thus $\mathfrak{H C}$ is a hereditary radical class. (See e.g. [5, Theorem 1, p. 4].)

Now suppose $J \in \mathcal{F}$ with $J$ an ideal of an ideal $I$ of some $R \in W$ with $J^{\prime}$ the ideal of $R$ generated by $J$. Then $J \in \mathcal{H}_{\alpha}$ for some $\alpha$ and so $J^{\prime} \in \mathcal{G H}_{\alpha}$. But then $J^{\prime} \in \mathscr{L G G H C}_{\alpha}=\mathcal{H}_{\alpha+1} \subseteq \mathcal{H}$. Therefore $\mathcal{H}$ is a strongly hereditary radical class containing $P$.

Finally, let $P \subseteq R$ where $R$ is a strongly hereditary radical class. Then $\mathfrak{H}_{1}=\beta \subseteq R$ and we proceed by induction assuming $\mathfrak{H}_{\alpha} \subseteq R$ for all $\alpha<\beta$. Then certainly $\mathcal{H}_{\beta} \subseteq \Omega$ when $\beta$ is a limit ordinal. Otherwise $\mathfrak{H}_{\beta-1} \subseteq R$ so, since $R$ has property (a), $\mathcal{G H}_{\beta-1} \subseteq \mathcal{G} R=R$. But $R$ is a hereditary radical and thus $\mathfrak{H C}_{\beta}=\mathcal{L I g G C}_{\beta-1} \subseteq \mathscr{L} \mathscr{I} R=\mathscr{L} R=R$. Hence $\mathfrak{H C}$ is the unique minimal strongly hereditary radical class containing $P$.

Writing $\mathfrak{H} \odot$ for the class $\mathfrak{H}$ just constructed, we can then state

COROLlaRy. Every subclass $\mathfrak{T}$ of an arbitrary universal class $\mathfrak{W}$ is:

(a) contained in a unique minimal radical class $2 \mathfrak{T}$,

(b) contained in a unique minimal hereditary radical class $29 \mathfrak{T}$,

(c) contained in a unique minimal strongly hereditary radical class TESIT.

\section{REFERENCES}

1. T. Anderson, N. Divinsky and A. Suliński, Hereditary radicals in associative and alternative rings, Canad. J. Math. 17 (1965), 594-603. 
2. E. P. Armendariz, Closure properties in radical theory, Pacific J. Math. 26 (1968), 1-7.

3. W. G. Leavitt and E. P. Armendariz, Nonhereditary semisimple classes, Proc. Amer. Math. Soc. 18 (1967), 1114-1117.

4. A. E. Hoffman and W. G. Leavitt, Properties inherited by the lower radical, Portugal Math. (to appear).

5. N. J. Divinsky, Rings and radicals, Univ. of Toronto Press, Ontario, 1965.

UNIVERSITY OF NEBRASKA 